\title{
TAYLOR SERIES WITH LIMIT-POINTS ON A FINITE NUMBER OF CIRCLES
}

\author{
EMMANUEL S. KATSOPRINAKIS
}

\begin{abstract}
Let $S(z): \sum_{n=0}^{\infty} a_{n} z_{n}$ be a power series with complex coefficients. For each $z$ in the unit circle $T=\{z \in \mathbb{C}:|z|=1\}$ we denote by $L(z)$ the set of limit-points of the sequence $\left\{s_{n}(z)\right\}$ of the partial sums of $S(z)$. In this paper we examine Taylor series for which the set $L(z)$, for $z$ in an infinite subset of $T$, is the union of a finite number, uniformly bounded in $z$, of concentric circles. We show that, if in addition $\liminf \left|a_{n}\right|>0$, a complete characterization of these series in terms of their coefficients is possible (see Theorem 1).
\end{abstract}

\section{INTRODUCTION}

Starting with a well-known theorem of Marcinkiewicz and Zygmund (see [4, 6, Vol. II, p. 178] or [1]) S. K. Pichorides suggested a research line aiming at a characterization of rational functions by geometric properties of the sequence of the partial sums of their Taylor expansions. Results in this direction have been obtained in $[2,3$, and 5]. The main result of this article is the following theorem:

Theorem 1. Let $S(z): \sum_{n=0}^{\infty} a_{n} z^{n}$ be a power series satisfying $\lim \inf \left|a_{n}\right|>0$ and let $E$ be an infinite subset of the unit circle $T=\{z \in \mathbb{C}:|z|=1\}$. Assume that there is a positive integer $M$, such that, for every $z$ in $E$, the set $L(z)$ of all limit points of the sequence $\left\{s_{n}(z)\right\}$ of the partial sums of $S(z)$ is the union of $M(z), 1 \leq M(z) \leq M$, concentric circles, $C_{1}(z), C_{2}(z), \ldots, C_{M(z)}(z)$, in the complex plane. Then, there are a positive integer $m$, a finite subset $\Omega$ of $T$, $m$ positive numbers $c_{0}, \ldots, c_{m-1}$, and $m$ real numbers $\vartheta_{0}, \ldots, \vartheta_{m-1}$, such that:

(i) $\lim \left|a_{m n+v}\right|=c_{v}>0, n \rightarrow \infty, v=0,1,2, \ldots, m-1$.

(ii) If $a_{n}=\left|a_{n}\right| \exp \left(i \varphi_{n}\right)$, then, $\lim \left(\varphi_{m n+v+1}-\varphi_{m n+v}\right)=\vartheta_{v}, n \rightarrow \infty$, $v=0,1,2, \ldots, m-1$.

(iii) If $\vartheta=\vartheta_{0}+\vartheta_{1}+\cdots+\vartheta_{m-1}$, then for each $z$ in $E-\Omega$ the series $\sum_{n=0}^{\infty}\left(a_{n}-a_{n-m} e^{i \theta}\right) z^{n}$ converges, where $a_{n}=0$ for $n<0$.

V. N. Nestoridis proved in [5] the case $M(z)=1$ of this theorem. In the same paper he also proved that conditions (i), (ii), and (iii) imply that the set $L(z)$ is the union of at most $m$ distinct concentric circles in the complex plane.

Received by the editors February 20, 1991.

1980 Mathematics Subject Classification (1985 Revision). Primary 30B10; Secondary 42A99.

Key words and phrases. Partial sums, limit-points, Taylor series. 
The proof of our Theorem 1 follows different lines than those in [5]. Roughly speaking we generate from the given series $S(z)$ some new series with the interesting property that they belong to the class of Taylor series for which a complete characterization has been obtained in [2 and 3]. We shall call these series "the limit-series of $S(z)$ "; the precise definition is given in $\S 3$, where we also prove some of their properties, which we shall need below. We shall also need the following:

Lemma 1. Under the hypotheses of Theorem 1 there are an integer $m, m \geq 1, a$ partition of the set $\mathbb{N}$ of natural numbers in $m$ sequences $\left\{\lambda_{n, 1}\right\},\left\{\lambda_{n, 2}\right\}, \ldots$, $\left\{\lambda_{n, m}\right\}, m$ positive numbers $c_{1}, c_{2}, \ldots, c_{m}$ and $m$ complex sequences $\left\{q_{k, 1}\right\}$, $\left\{q_{k, 2}\right\}, \ldots,\left\{q_{k, m}\right\}$, where, for $j \neq \rho$, either $c_{j} \neq c_{\rho}$ or $\left\{q_{k, j}\right\} \neq\left\{q_{k, \rho}\right\}$, such that for $j=1,2, \ldots, m$,

$$
\lim _{n}\left|a_{\lambda_{n, j}}\right|=c_{j} \quad \text { and } \quad \lim _{n} \frac{a_{\lambda_{n, j}+k}}{a_{\lambda_{n, j}}}=q_{k, j}, \quad k=1,2,3, \ldots
$$

The proof of Theorem 1 is organized as follows: First, in $\S 2$ we take for granted Lemma 1 and prove the theorem. Next, in $\S 3$, by making essential use of the results in [2 and 3], we prove the above mentioned properties of the "limit-series of $S(z)$." Finally, in $\S 4$ using these properties, we prove Lemma 1. In the last paragraph 5 we offer some comments.

\section{ProOF OF THEOREM 1}

In this paragraph we take for granted Lemma 1 . We shall show that there is a positive integer $n_{0}$ such that for $n \geq n_{0}$ the sequences $\left\{\lambda_{n, j}\right\}, j=1, \ldots, m$, in this lemma, are arithmetic progressions with common ratio $m$. Then, parts (i) and (ii) of Theorem 1 are immediate consequences of (1) (reenumerating if necessary the numbers $\left.c_{1}, c_{2}, \ldots, c_{m}\right)$.

We consider the sequence $\left\{\lambda_{n, 1}+1\right\}$. By (1) we have

$$
\lim _{n}\left|a_{\lambda_{n, 1}+1}\right|=c_{1}\left|q_{1,1}\right| \quad \text { and } \lim _{n} \frac{a_{\lambda_{n, 1}+1+k}}{a_{\lambda_{n, 1}+1}}=\frac{q_{k+1,1}}{q_{1,1}}, \quad k=1,2,3, \ldots
$$

Lemma 1 shows that the set $\left\{\lambda_{n, 1}+1\right\}$ cannot have infinite intersections with two of the sets $\left\{\lambda_{n, j}\right\}, j=1,2, \ldots, m$. Since $\left\{\lambda_{n, j}\right\}, j=1,2, \ldots, m$, form a partition of $\mathbb{N}$, if $m>1$, infinitely many terms of $\left\{\lambda_{n, 1}+1\right\}$ do not belong to $\left\{\lambda_{n, 1}\right\}$. Thus, there is an integer $n_{1} \geq 0$, such that the sequence $\left\{\lambda_{n+n_{1}, 1}+1\right\}$ must be a subset of one and only one of $\left\{\lambda_{n, 2}\right\}, \ldots,\left\{\lambda_{n, m}\right\}$. Assume that we have enumerated the $\left\{\lambda_{n, j}\right\}$ 's in such a way that $\left\{\lambda_{n+n_{1}, 1}+1\right\}$ is a subset of $\left\{\lambda_{n, 2}\right\}$.

If $m>2$, and since $\left\{\lambda_{n+n_{1}, 1}+2\right\}$ is a subset of $\left\{\lambda_{n, 2}+1\right\}$, an analogous argument and $a$, possibly, further reenumeration shows that there is an integer $n_{2} \geq 0$, such that $\left\{\lambda_{n+n_{2}, 2}+1\right\}$ is a subset of $\left\{\lambda_{n, 3}\right\}$. So for every natural number $n$ we have

$$
\left\{\lambda_{n+n_{1}+n_{2}, 1}+2\right\} \subset\left\{\lambda_{n+n_{2}, 2}+1\right\} \subset\left\{\lambda_{n, 3}\right\} .
$$

Continuing in the same way we conclude that there are $m$ natural numbers $n_{1}, n_{2}, \ldots, n_{m}$, such that for all $n$ we have

$\left\{\lambda_{n+n_{1}+\cdots+n_{m}, 1}+m\right\} \subset\left\{\lambda_{n+n_{2}+\cdots+n_{m}, 2}+m-1\right\} \subset \cdots \subset\left\{\lambda_{n+n_{m}, m}+1\right\} \subset\left\{\lambda_{n, 1}\right\}$. 
We write $n_{0}=n_{1}+n_{2}+\cdots+n_{m}$. Then, $\left\{\lambda_{n+n_{0}, 1}+m\right\}$ is a subset of $\left\{\lambda_{n, 1}\right\}$. From $\left\{\lambda_{n+n_{2}+\cdots+n_{m}, 2}+m-1\right\} \subset\left\{\lambda_{n, 1}\right\}$ we obtain

$$
\left\{\lambda_{n+\left(n_{0}-n_{1}\right), 2}+m-1\right\} \subset\left\{\lambda_{n, 1}\right\}
$$

and hence $\left\{\lambda_{n+n_{0}, 2}+m\right\} \subset\left\{\lambda_{n+n_{1}, 1}+1\right\} \subset\left\{\lambda_{n, 2}\right\}$. Continuing in the same way we obtain the inclusions

$$
\left\{\lambda_{n+n_{0}, j}+m\right\} \subset\left\{\lambda_{n, j}\right\}, \quad j=1,2, \ldots, m, n \in \mathbb{N} .
$$

Since the sequence $\left\{\lambda_{n, j}\right\}, j=1,2, \ldots, m$, are strictly increasing, we shall also have for every $n$ in $\mathbb{N}$,

$$
\left\{\lambda_{n+n_{0}, j}+m\right\} \subset\left\{\lambda_{n+n_{0}, j}\right\}, \quad j=1,2, \ldots, m .
$$

The last inclusions combined with the fact that the $\left\{\lambda_{n, j}\right\}, j=1,2, \ldots, m$, form a partition of $\mathbb{N}$ complete the proof of (i) and (ii) of Theorem 1 .

It remains to prove (iii). In the course of the proof we shall need to remove from $E$ a finite set $\Omega=\Omega_{1} \cup \Omega_{2}$. The sets $\Omega_{1}, \Omega_{2}$ will be defined in terms of $c_{j}, \vartheta_{j}, j=0,1, \ldots, m-1$, whose existence has just been proved. $\Omega_{1}$ will be the set $\left\{z \in T: 1-e^{i \theta} z^{m}=0\right\} \quad\left(\vartheta=\vartheta_{0}+\vartheta_{1}+\cdots+\vartheta_{m-1}\right)$ and $\Omega_{2}$ the set of $z$ 's for which the partial sums of order less than $2(m M+1)$ of the series $\sum d_{k} z^{k}$ are pairwise distinct, where $d_{m k+\nu}=d_{\nu} \exp (i k \vartheta), k=$ $1,2, \ldots, \nu=0,1, \ldots, m-1, d_{0}=c_{0}, d_{\nu}=c_{\nu} \exp \left(\vartheta_{0}+\vartheta_{1}+\cdots+\vartheta_{\nu}-\nu t\right) i$, $\nu=1,2, \ldots, m-1$, and $m t=\vartheta$. For $z$ in $E-\Omega$ we write

$$
\sigma_{N}(z)=\frac{1}{1-e^{i \theta} z^{m}} \sum_{k=0}^{N}\left(a_{k}-a_{k-m} e^{i \theta}\right) z^{k},
$$

where $a_{k}=0$ if $k<0$. Under the hypotheses of Theorem 1 we have that, for every $z$ in $E$, the sequence $\left\{s_{N}(z)\right\}$ of the partial sums of $S(z)$ is bounded and so, for every $z$ in $E-\Omega,\left\{\sigma_{N}(z)\right\}$ is also bounded. Moreover, for every $z$ in $E-\Omega$, we shall show that $\lim \sigma_{N}(z)$ exists and

$$
\lim \sigma_{N}(z)=\sigma(z), \quad N \rightarrow \infty,
$$

where $\sigma(z)$ is the common center of the concentric circles $C_{j}(z), j=1,2, \ldots$, $M(z)$. This means that

$$
\sigma(z)=\frac{1}{1-e^{i \theta} z^{m}} \sum_{k=0}^{\infty}\left(a_{k}-a_{k-m} e^{i \theta}\right) z^{k}, \quad z \in E-\Omega,
$$

which implies (iii) and completes the proof of Theorem 1.

In order to prove (2) it suffices to show that for each $z$ in $E-\Omega$ and each $v$ in $\{0,1, \ldots, m-1\}$ we have

$$
\lim _{\nu} \sigma_{m\left(\lambda_{\nu}+1\right)+v-1}(z)=\sigma(z),
$$

for every subsequence $\left\{\lambda_{\nu}\right\}$ of $\mathbb{N}$ for which this limit exists.

We fix $z \in E-\Omega$ and $v \in\{0,1, \ldots, m-1\}$. Let $\left\{\lambda_{\nu}\right\}$ be a subsequence of $\mathbb{N}$ and $t(z)=t\left(z, v,\left\{\lambda_{\nu}\right\}\right)$ a complex number, such that

$$
\lim _{\nu} \sigma_{m\left(\lambda_{\nu}+1\right)+v-1}(z)=t(z) .
$$

We have to show that $t(z)=\sigma(z)$. 
Since the sequences $\left\{a_{n}\right\},\left\{s_{n}(z)\right\},\left\{z^{n}\right\}$ are bounded, there is a subsequence of $\left\{\lambda_{\nu}\right\}$, which for simplicity we denote again by $\left\{\lambda_{\nu}\right\}$, a real number $\varphi=\varphi\left(v,\left\{\lambda_{\nu}\right\}\right)$ and two complex numbers $\tau(z)=\tau\left(z, v,\left\{\lambda_{\nu}\right\}\right)$ and $\xi(z)=$ $\xi\left(z, v,\left\{\lambda_{\nu}\right\}\right)$, where $|\xi(z)|=1$, such that

(4) $\quad \lim _{\nu} \operatorname{Arg} a_{m \lambda_{\nu}+v}=\varphi, \quad \lim _{\nu} s_{m \lambda_{\nu}+v-1}(z)=\tau(z), \quad \lim _{\nu} z^{m \lambda_{\nu}+v}=\xi(z)$.

Using (i), (ii) and (4) we define a sequence $\left\{b_{n}\right\}$ of complex numbers as follows:

$$
\begin{aligned}
& b_{0}=c_{v} \exp (i \varphi)=\lim _{\nu} a_{m \lambda_{\nu}+v} \\
& b_{1}=c_{v+1} \exp \left[i\left(\varphi+\vartheta_{v}\right)\right]=\lim _{\nu} a_{m \lambda_{\nu}+v+1} \\
& \vdots \quad \vdots \quad \vdots \\
& b_{m-v-1}=c_{m-1} \exp \left[i\left(\varphi+\vartheta_{v}+\cdots+\vartheta_{m-2}\right)\right]=\lim _{\nu} a_{m \lambda_{\nu}+m-1} \\
& b_{m-v}=c_{0} \exp \left[i\left(\varphi+\vartheta_{v}+\cdots+\vartheta_{m-1}\right)\right]=\lim _{\nu} a_{m \lambda_{\nu}+m} \\
& b_{m-v+1}=c_{1} \exp \left[i\left(\varphi+\vartheta_{v}+\cdots+\vartheta_{m-1}+\vartheta_{0}\right)\right]=\lim _{\nu} a_{m \lambda_{\nu}+m+1} \\
& \vdots \quad \vdots \quad \vdots \\
& b_{m-1}=c_{v-1} \exp \left[i\left(\varphi+\vartheta-\vartheta_{v-1}\right)\right]=\lim _{\nu} a_{m \lambda_{\nu}+v+m-1}
\end{aligned}
$$

and for $n=m k+\rho, k=0,1,2, \ldots, \rho=0,1, \ldots, m-1, b_{n}=b_{\rho} \exp (i k \vartheta)$ (we recall that $\vartheta=\vartheta_{0}+\vartheta_{1}+\cdots+\vartheta_{m-1}$ ). Using again (i) and (ii) of the theorem we obtain

$$
b_{n}=b_{m k+\rho}=b_{\rho} \exp (i k \vartheta)=\lim _{\nu} a_{m \lambda_{\nu}+v+m k+\rho} .
$$

We consider now the series

$$
S\left(\left\{\lambda_{\nu}\right\}\right): \sum_{n=0}^{\infty} b_{n} z^{n}=A(z) \sum_{k=0}^{\infty}\left(e^{i \theta} z^{m}\right)^{k},
$$

where $A(z)=b_{0}+b_{1} z+\cdots+b_{m-1} z^{m-1}$ (as we shall see in $\S 3$ this series is one of the "limit-series of $S(z)$ "). An easy computation shows that the partial sums $s_{n}^{\prime}(z)$ of $S\left(\left\{\lambda_{\nu}\right\}\right)$ lie on a finite number of concentric circles centered at the point $\sigma_{1}(z)=\sigma_{1}\left(z, v,\left\{\lambda_{\nu}\right\}\right)=A(z) /\left(1-e^{i \theta} z^{m}\right)$ (see also (10), Theorem $2, \S 3)$. On the other hand, we shall see in a moment that

$$
\sigma_{1}(z)=\frac{\sigma(z)-\tau(z)}{\xi(z)}
$$

and

$$
\frac{t(z)-\tau(z)}{\xi(z)}=\frac{A(z)}{1-e^{i \theta} z^{m}}=\sigma_{1}(z),
$$

which imply the desired result $t(z)=\sigma(z)$. Thus, it remains to prove (6) and (7).

From (4), (5) and the relation

$$
s_{m \lambda_{\nu}+v+n}(z)-s_{m \lambda_{\nu}+v-1}(z)=z^{m \lambda_{\nu}+v}\left(a_{m \lambda_{\nu}+v}+\cdots+a_{m \lambda_{\nu}+v+n} z^{n}\right),
$$


we see that $\lim _{\nu} s_{m \lambda_{\nu}+v+n}(z)$ exists, for every $n=0,1,2, \ldots$. We call this limit $\tau_{n}(z)$ and on observing that $s_{n}^{\prime}(z)=\lim _{\nu}\left(a_{m \lambda_{\nu}+v}+\cdots+a_{m \lambda_{\nu}+v+n} z^{n}\right)$, we have

$$
s_{n}^{\prime}(z)=\frac{\tau_{n}(z)-\tau(z)}{\xi(z)} .
$$

But, $\tau_{n}(z) \in L(z)$, for every $n=0,1,2, \ldots$. Thus, for each $n$ in $\mathbb{N}$, there is one circle $C_{j}(z), j=1,2, \ldots, M(z)$, of radius say $r_{n}(z)$, such that $\left|\tau_{n}(z)-\sigma(z)\right|=r_{n}(z)$. Now, for each $n$ in $\mathbb{N}$, we have

$$
\left|s_{n}^{\prime}(z)-\frac{\sigma(z)-\tau(z)}{\xi(z)}\right|=\left|\tau_{n}(z)-\sigma(z)\right|=r_{n}(z),
$$

since $|\xi(z)|=1$. On observing that the series $S\left(\left\{\lambda_{\nu}\right\}\right)$ differs from $\sum d_{k} z^{k}$ by a constant factor $e^{i \varphi}$ and that $z \notin \Omega_{2}$ we can easily see that at least one of the circles $C_{j}(z), j=1, \ldots, M(z)$, has three points in common with one of the limit circles of the partial sums of $S\left(\left\{\lambda_{\nu}\right\}\right)$. This proves $(6)$.

Finally, (7) is an immediate consequence of (3), (4), (5) and the obvious relation

$$
\frac{\sigma_{m \lambda_{\nu}+v+m-1}(z)-s_{m \lambda_{\nu}+v-1}(z)}{z^{m \lambda_{\nu}+v}}=\frac{a_{m \lambda_{\nu}+v}+\cdots+a_{m \lambda_{v}+v+m-1} z^{m-1}}{1-e^{i \theta} z^{m}} .
$$

This completes the proof of Theorem 1 .

\section{THE LIMIT-SERIES OF $S(z)$}

In this paragraph we shall give the precise definition of the "limit-series of $S(z)$ " and we shall prove three properties of these series on which the proof of Lemma 1 is based.

In the course of the proofs we shall need some results from [2 and 3]. In order to facilitate the reading we collect these results in the following

Theorem 2. Let $S(z): \sum_{n=0}^{\infty} a_{n} z^{n}$ be a power series with complex coefficients. Then, (a), (b) and (c) are equivalent,

(a) For every $z$ in a nondenumerable subset $E$ of the unit circle $T$, the partial sums

$$
s_{n}(z)=\sum_{k=0}^{n} a_{k} z^{k}
$$

of $S(z)$, lie on the union of a finite number $M(z)$ of circles, $C_{1}(z), C_{2}(z), \ldots$, $C_{M(z)}(z)$, in the complex plane.

(b) Same as (a), but the numbers $M(z), z \in E$, are uniformly bounded in $z$, i.e. there is a positive integer $M$, such that $1 \leq M(z) \leq M, z \in E$. In this case we may assume that $E$ is infinite (not necessarily nondenumerable).

(c) The series $S(z)$ has a representation of the form

$$
\sum_{n=0}^{\infty} a_{n} z^{n}=P(z)+z^{k} A(z) \sum_{n=0}^{\infty}\left(e^{i \theta} z^{\mu}\right)^{n},
$$

where $\vartheta \in[0,2 \pi), k, \mu$ are integers, $k \geq 0,1 \leq \mu \leq 4(2 M+1)^{2} M^{2 M+1}$, $P(z)=a_{0}+a_{1} z+\cdots+a_{k-1} z^{k-1}$ if $k \geq 1$, or $P(z) \equiv 0$ if $k=0$, and $A(z)=a_{k}+a_{k+1} z+\cdots a_{k+\mu-1} z^{k+\mu-1}$. 
Moreover, under the above conditions there are a finite subset $\Omega$ of the unit circle $T$ and $\mu$ concentric circles (not necessarily distinct) $C_{1}^{\prime}(z), \ldots, C_{\mu}^{\prime}(z)$, among the $C_{j}(z), j=1,2, \ldots, M(z)$, such that, for every $z$ in $E-\Omega$ the partial sums of $S(z)$, with indices $\geq k$, satisfy

$$
\begin{aligned}
& s_{\mu n+\mu-1}(z) \in C_{1}^{\prime}(z) \\
& s_{\mu n+\mu-2}(z) \in C_{2}^{\prime}(z) \\
& s_{\mu n}(z) \in C_{\mu}^{\prime}(z) .
\end{aligned}
$$

The common center of these circles is given by

$$
P(z)+z^{k} \frac{A(z)}{1-e^{i \theta} z^{\mu}},
$$

and if $d_{0}=a_{k}, d_{\nu}=a_{k+\nu} \exp (-i \nu t), \nu=1,2,3, \ldots, \mu-1$, where $\mu t=\vartheta$, then, the radii of these circles are respectively

$$
R_{v}(z)=\left|\frac{A_{v}(\zeta)}{1-\zeta^{\mu}}\right|, \quad v=0,1,2, \ldots, \mu-1,
$$

where $\zeta=z \exp (i t)$ and $A_{v}(\zeta)$ are the polynomials obtained by cyclic permutations of the coefficients of the polynomial $A_{0}(\zeta)=d_{0}+d_{1} \zeta+\cdots+d_{\mu-1} \zeta^{\mu-1}$, i.e. $A_{v}(\zeta)=d_{v}+d_{v+1} \zeta+\cdots+d_{v-1} \zeta^{\mu-1}$.

We proceed now to the definition of the limit-series of $S(z)$.

From the assumptions of Theorem 1 we know that the coefficients of $S(z)$ satisfy $\lim \inf \left|a_{n}\right|>0$ and it is easy to see that the sequence $\left\{a_{n}\right\}$ is bounded. Thus, every limit-point of $\left\{a_{n}\right\}$ is finite and different from zero.

Let $b_{0}$ be such a limit-point of $\left\{a_{n}\right\}$. Then, there is a subsequence $\left\{a_{\lambda_{\nu}}\right\}$ of $\left\{a_{n}\right\}$ such that $\lim _{\nu} a_{\lambda_{\nu}}=b_{0}$. Using a diagonal argument we may assume that all the sequences $\left\{a_{\lambda_{\nu}+k}\right\}, k=0, \pm 1, \pm 2, \ldots$, converge. Thus, there are $b_{k}$, such that

$$
\lim _{\nu} a_{\lambda_{\nu}+k}=b_{k}, \quad \text { for each } k=0, \pm 1, \pm 2, \ldots
$$

Definition. For each sequence $\left\{\lambda_{\nu}\right\}$ as above, we define the "limit-series" of $S(z)$, which corresponds to $\left\{\lambda_{\nu}\right\}$, to be the series

$$
S\left(\left\{\lambda_{\nu}\right\}\right): \sum_{k=0}^{\infty} b_{k} z^{k} .
$$

We prove now three properties of the limit-series of $S(z)$, which we shall use in $\S 4$. These properties are rather deep and their proof depends heavily on Theorem 2.

Property 1. Let $N_{0}=4(2 M+1)^{2} M^{2 M+1}$, where $M$ is the number given in Theorem 1. Then, there are integer $\mu, 1 \leq \mu \leq N_{0}$, and a real number $\vartheta \in$ $[0,2 \pi)$, such that the limit-series $S\left(\left\{\lambda_{\nu}\right\}\right)$ has a representation of the form

$$
\left(b_{0}+b_{1} z+\cdots+b_{\mu-1} z^{\mu-1}\right) \sum_{n=0}^{\infty}\left(e^{i \theta} z^{\mu}\right)^{n} .
$$


Proof. (Compare with the proof of part (iii) of Theorem 1.)

Let $E_{0}$ be an infinite denumerable subset of $E$. Using diagonal arguments we can find a subsequence of $\left\{\lambda_{\nu}\right\}$, which for simplicity we denote again by $\left\{\lambda_{\nu}\right\}$, such that, for every $z$ in $E_{0}$, the $\operatorname{limits}_{\lim _{\nu}} z^{\lambda_{\nu}}$ and $\lim _{\nu} s_{\lambda_{\nu}-1}(z)$ exist, where $s_{n}(z)=\sum_{k=0}^{n} a_{k} z^{k}$. We write

$$
\lim _{\nu} s_{\lambda_{\nu}-1}(z)=\tau(z) \quad \text { and } \quad \lim _{\nu} z^{\lambda_{\nu}}=\xi(z), \quad \text { where }|\xi(z)|=1 .
$$

By (12), (14) and the relation

$$
s_{\lambda_{\nu}+n}(z)-s_{\lambda_{\nu}-1}(z)=z^{\lambda_{\nu}}\left(a_{\lambda_{\nu}}+a_{\lambda_{\nu}+1} z+\cdots+a_{\lambda_{\nu}+n} z^{n}\right),
$$

we have, for every $z$ in $E_{0}$, that

$$
\lim _{\nu} s_{\lambda_{\nu}+n}(z)=\tau_{n}(z), \quad n=0,1,2,3, \ldots,
$$

where $\tau_{n}(z)=\tau(z)+\xi(z)\left(b_{0}+b_{1} z+\cdots+b_{n} z^{n}\right)$. Hence, writing $s_{n}^{\prime}(z)$ for the partial sums $b_{0}+b_{1} z+\cdots+b_{n} z^{n}$ of $S\left(\left\{\lambda_{\nu}\right\}\right)$, we obtain

$$
s^{\prime}(z)=\left[\tau_{n}(z)-\tau(z)\right] / \xi(z),
$$

for every $z$ in $E_{0}$ and $n=0,1,2,3, \ldots$.

Now, for every $z$ in $E_{0}$ and $n=0,1,2, \ldots, \tau_{n}(z) \in L(z)$ and so, if $\sigma(z)$ is the common center of the circles $C_{j}(z), j=1,2,3, \ldots, M(z)$, there is one of these circles of radius, say $r_{n}(z)$, such that, $\left|\tau_{n}(z)-\sigma(z)\right|=r_{n}(z)$. Using this relation, (15) and the fact that $|\xi(z)|=1$, we obtain that

$$
\left|s_{n}^{\prime}(z)-[\xi(z)]^{-1}[\sigma(z)-\tau(z)]\right|=r_{n}(z) .
$$

This means that the partial sums of $S\left(\left\{\lambda_{v}\right\}\right)$ lie on the union of a finite number, aniformly bounded in $z$, of circles centered at the point $\sigma_{1}(z)=$ $[\sigma(z)-\tau(z)] / \xi(z)$, for every $z$ in $E_{0}$. Since $E_{0}$ is infinite, Theorem 2 implies that the series $S\left(\left\{\lambda_{\nu}\right\}\right)$ has a representation of the form (8).

In order to see that this representation coincides with (13) it suffices to show that its nonperiodic part $P(z)$ is identically zero (i.e. $k=0$ in (8)). To prove this we consider the power series

$$
\sum_{k=1}^{\infty} b_{-k} w^{k}
$$

The partial sums $s_{n}^{\prime \prime}(w)$ of (17) for $w=z^{-1}, z$ in $E_{0}$, satisfy the following relation (compare with $(15)), s_{n}^{\prime \prime}(w)=\left[\tau(z)-\tau_{-(n+1)}(z)\right] / \xi(z)$, where $\tau_{-(n+1)}(z) \in L(z)$, for every $z$ in $E_{0}$ and $n=0,1,2, \ldots$ Indeed, for $w=z^{-1}, z$ in $E_{0}$, using (12) and (14) we have

$$
\begin{aligned}
s_{n}^{\prime \prime}(w) & =b_{-1} z^{-1}+\cdots+b_{-n} z^{-n}=\lim _{\nu}\left(a_{\lambda_{\nu}-1} z^{-1}+\cdots+a_{\lambda_{\nu}-n} z^{-n}\right) \\
& =\lim _{\nu} z^{-\lambda_{\nu}}\left(a_{\lambda_{\nu}-1} z^{\lambda_{\nu}-1}+\cdots+a_{\lambda_{\nu}-n} z^{\lambda_{\nu}-n}\right) \\
& =\lim _{\nu} z^{-\lambda_{\nu}}\left(s_{\lambda_{\nu}-1}(z)-s_{\lambda_{\nu}-n-1}(z)\right) \\
& =\left[\tau(z)-\tau_{-(n+1)}(z)\right] / \xi(z) .
\end{aligned}
$$

Since $\tau_{-(n+1)}(z) \in L(z)$, for every $z$ in $E_{0}$ and $n=0,1,2, \ldots$, we see, exactly as in the case of $s_{n}^{\prime}(z)$, that the partial sums $s_{n}^{\prime \prime}(w)$ of (17) lie on 
the union of a finite number, uniformly bounded in $z$, of circles centered at the point $\sigma_{2}(w)=\sigma_{2}\left(z^{-1}\right)=[\tau(z)-\sigma(z)] / \xi(z)$, for every $z$ in $E_{0}$, and so, Theorem 2 applies to the series (17). Moreover, we observe that for every $z$ in $E_{0}$ we have

$$
\sigma_{1}(z)=-\sigma_{2}\left(z^{-1}\right) .
$$

On the other hand, Theorem 2 gives another formula for each of these centers. As we shall see in a moment these formulae and (18) will yield the desired vanishing of the nonperiodic part $P(z)$ of the representation of $S\left(\left\{\lambda_{\nu}\right\}\right)$. It will simplify the exposition of the proof if we rewrite the representations given by (8), for the series $S\left(\left\{\lambda_{\nu}\right\}\right)$ and (17), as follows:

$$
\begin{aligned}
& \sum_{n=0}^{\infty} b_{k} z^{k}=P(z)+z^{\mu} A(z) \sum_{n=0}^{\infty}\left(e^{i \theta} z^{\mu}\right)^{n} \\
& \sum_{n=1}^{\infty} b_{-k} w^{k}=Q(w)+w^{\mu} B(w) \sum_{n=0}^{\infty}\left(e^{i \theta^{\prime}} w^{\mu}\right)^{n},
\end{aligned}
$$

where

$$
\begin{gathered}
P(z)=b_{0}+b_{1} z+\cdots+b_{\mu-1} z^{\mu-1} \\
A(z)=b_{\mu}+b_{\mu+1} z+\cdots+b_{2 \mu-1} z^{\mu-1} \\
Q(w)=b_{-1} w+b_{-2} w^{2}+\cdots+b_{-(\mu-1)} w^{\mu-1}
\end{gathered}
$$

and

$$
B(w)=b_{-\mu}+b_{-(\mu+1)} w+\cdots+b_{-(2 \mu-1)} w^{\mu-1} .
$$

In other words, we chose the integer $\mu$ sufficiently large (e.g. a suitable multiple of the periods of the series under consideration), so that the polynomials $P, A, Q, B$ have the same degree $\mu-1$. Now removing from $E_{0}$, as in the proof of part (iii) of Theorem 1, a finite set $\Omega_{0}$ and using (10) of Theorem 2 we see that for every $z$ in $E_{0}^{\prime}=E_{0}-\Omega_{0}$ we have

$$
\sigma_{1}(z)=P(z)+z^{\mu} \frac{A(z)}{1-e^{i \theta} z^{\mu}}, \quad \sigma_{2}(w)=Q(w)+w^{\mu} \frac{B(w)}{1-e^{i \theta^{\prime}} w^{\mu}},
$$

for the centers corresponding to the series (19) and (20).

From these formulae and (18) we find

$$
\begin{aligned}
& P(z) z^{\mu-1}\left(1-e^{i \theta} z^{\mu}\right)\left(z^{\mu}-e^{i \theta^{\prime}}\right)+z^{2 \mu+1} A(z)\left(z^{\mu}-e^{i \theta^{\prime}}\right) \\
& \quad+z^{\mu-1} Q\left(z^{-1}\right)\left(z^{\mu}-e^{i \theta^{\prime}}\right)\left(1-e^{i \theta} z^{\mu}\right)+z^{\mu-1} B\left(z^{-1}\right)\left(1-e^{i \theta} z^{\mu}\right)=0,
\end{aligned}
$$

for every $z$ in $E_{0}^{\prime}$. The terms of degree $\mu-1,2 \mu-1$ and $3 \mu-1$ of (21) give respectively

$$
b_{-\mu}-e^{i \theta^{\prime}} b_{0}=0, \quad b_{0}-e^{i \theta^{\prime}} b_{\mu}-e^{i \theta}\left(b_{-\mu}-e^{i \theta^{\prime}} b_{0}\right)=0, \quad b_{\mu}-e^{i \theta} b_{0}=0 .
$$

Since $\vartheta, \vartheta^{\prime} \in[0,2 \pi)$ and $b_{0} \neq 0$ we conclude that $\vartheta^{\prime}=-\vartheta$. Substituting $\vartheta^{\prime}$ by $-\vartheta$ in (21), deleting the common factor $1-e^{i \theta} z^{\mu}$ and using the formulae of the polynomials $P, A, Q, B$, we obtain

$$
\sum_{k=-2 \mu+1}^{\mu-1}\left(b_{k} e^{i \theta}-b_{k+\mu}\right) z^{k+2 \mu-1}=0,
$$


for every $z$ in $E_{0}^{\prime}$. Thus,

$$
\begin{gathered}
b_{-(\mu-1)}=b_{-(2 \mu-1)} e^{i \theta}, b_{-(\mu-2)}=b_{-(2 \mu-2)} e^{i \theta}, \ldots, b_{0}=b_{-\mu} e^{i \theta}, \\
b_{1}=b_{-\mu+1} e^{i \theta}, \ldots, b_{\mu}=b_{0} e^{i \theta}, \ldots, b_{2 \mu-1}=b_{\mu-1} e^{i \theta},
\end{gathered}
$$

which proves Property 1.

The next property is an immediate consequence of Theorem 2 and Property 1.

Let $\left\{s_{\eta}^{\prime}(z)\right\}$ be the sequence of the partial sums of the limit-series $S\left(\left\{\lambda_{\nu}\right\}\right)$. Then we have

Property 2. For every infinite subset $E^{\prime}$ of $E$ there are an infinite subset $E_{1}$ of $E^{\prime}$ and an ordered $\mu$-tuple of indices $\left(j_{1}, j_{2}, \ldots, j_{\mu}\right), j_{k} \in\{1,2, \ldots, M\}$, $k=1,2, \ldots, \mu$ (the $j_{k}$ 's are not necessarily distinct), such that, for every $z$ in $E_{1}$ there are $\mu$ concentric circles $C_{1}^{\prime}(z), \ldots, C_{\mu}^{\prime}(z)$, with the following properties:

(a) For each $z$ in $E_{1}$ there is a Euclidean transformation (translation plus rotation) which sends $C_{j_{k}}(z)$ to $C_{k}^{\prime}(z)$ for all $k=1,2, \ldots, \mu$.

(b) For every $z$ in $E_{1}$ and $n=0,1,2, \ldots$, the sequence $\left\{s_{n}^{\prime}(z)\right\}$ satisfies the relations (9) of Theorem 2.

(c) If $d_{0}=b_{0}, d_{k}=b_{k} \exp (-i k t), k=1,2,3, \ldots, \mu-1$, where $\mu t=\vartheta$, then, the radii of these circles are given by the formulae (11) of Theorem 2, respectively.

Proof. If the sequence $\left\{s_{n}(z)\right\}$ of the partial sums of $S(z)$ is bounded for some $z$ in $T$, then, for this $z$, we must have the relations (14) and so (15) and (16). In particular, this happens when $z \in E$ and thus for every $z$ in $E^{\prime}$. We fix such a $z$. Theorem 2, the proof of Property 1 and in particular (16) imply that there is an ordered $\mu$-tuple $\left(j_{1}, j_{2}, \ldots, j_{\mu}\right), j_{k} \in\{1,2, \ldots, M\}$, $k=1, \ldots, \mu$ such that, for this $z$, Property 2 holds (we omit the easy proof). In order to prove Property 2 for all $z$ in an infinite subset $E_{1}$ of $E^{\prime}$, we simply observe that $E^{\prime}$ is infinite and the number of the above ordered $\mu$-tuples is finite.

The last property which we shall need in $\S 4$ refers to two limit-series of $S(z)$ and it is the following:

Property 3. If two limit-series of $S(z), S\left(\left\{\lambda_{\nu}\right\}\right): \sum_{n=0}^{\infty} b_{k} z^{k}$ and $S\left(\left\{\lambda_{\nu}^{\prime}\right\}\right)$ : $\sum_{n=0}^{\infty} b_{k}^{\prime} z^{k}$ have the same $\mu$-tuple $\left(j_{1}, j_{2}, \ldots, j_{\mu}\right)$ of indices, for infinitely many $z$ in $E$, then

(a) If $b_{0}^{\prime}=b_{0} \omega$, then either $|\omega|=1$ or $|\omega|^{2}=\left|b_{1} b_{3} / b_{0} b_{2}\right|$.

(b) If $|\omega|=1$ in (a), then $b_{k}^{\prime} / b_{0}^{\prime}=b_{k} / b_{0}$, for every $k=1,2,3, \ldots$.

Proof. From Property 1 we know that

$$
S\left(\left\{\lambda_{\nu}\right\}\right):\left(b_{0}+b_{1} z+\cdots+b_{\mu-1} z^{\mu-1}\right) \sum_{n=0}^{\infty}\left(e^{i \theta} z^{\mu}\right)^{n}, \quad \vartheta \in[0,2 \pi),
$$

and

$$
S\left(\left\{\lambda_{\nu}^{\prime}\right\}\right):\left(b_{0}^{\prime}+b_{1}^{\prime} z+\cdots+b_{\mu-1}^{\prime} z^{\mu-1}\right) \sum_{n=0}^{\infty}\left(e^{i \theta^{\prime}} z^{\mu}\right)^{n}, \quad \vartheta^{\prime} \in[0,2 \pi)
$$


Let $E^{\prime}$ be an infinite subset of $E$, such that, for every $z$ in $E^{\prime}$, the above limit-series have the same $\mu$-tuple of indices. Then, (c) of Property 2 gives that

$$
\left|\frac{A_{v}(\zeta)}{1-\zeta^{\mu}}\right|=\left|\frac{A_{v}^{\prime}\left(\zeta^{\prime}\right)}{1-\left(\zeta^{\prime}\right)^{\mu}}\right|, \quad v=0,1,2, \ldots, \mu-1,
$$

where $\zeta=z \exp (i t), \zeta^{\prime}=z \exp \left(i t^{\prime}\right),\left(\mu t=\vartheta, \mu t^{\prime}=\vartheta^{\prime}\right), z \in E^{\prime}$.

For $v=0,(22)$ becomes

$$
\begin{aligned}
& \left|1-z^{\mu} \exp \left(i \vartheta^{\prime}\right)\right|\left|b_{0}+b_{1} z+\cdots+b_{\mu-1} z^{\mu-1}\right| \\
& \quad=\left|1-z^{\mu} \exp (i \vartheta)\right|\left|b_{0}^{\prime}+b_{1}^{\prime} z+\cdots+b_{\mu-1}^{\prime} z^{\mu-1}\right|,
\end{aligned}
$$

for every $z \in E^{\prime}$. Squaring (23) and comparing the terms of degree $-\mu$ we obtain

$$
\left(\left|b_{0}\right|^{2}+\cdots+\left|b_{\mu-1}\right|^{2}\right) \exp (i \vartheta)=\left(\left|b_{0}^{\prime}\right|^{2}+\cdots+\left|b_{\mu-1}\right|^{2}\right) \exp \left(i \vartheta^{\prime}\right)
$$

which in turn gives $\vartheta^{\prime}=\vartheta$ (we recall that $\vartheta, \vartheta^{\prime} \in[0,2 \pi)$ ).

Further, since then $\zeta=\zeta^{\prime},(22)$ yields

$$
\left|A_{v}(\zeta)\right|=\left|A_{v}^{\prime}(\zeta)\right|, \quad v=0,1,2, \ldots, \mu-1,
$$

for every $z$ in $E^{\prime}\left(\zeta=z e^{i t}, \mu t=\vartheta\right)$. Each one of (24) gives a number of relations between the coefficients $d_{j}$ and $d_{j}^{\prime}, j=0,1, \ldots, \mu-1$, (compare with Lemma 1 of [2]). From these relations we shall need only the following:

$$
\begin{gathered}
\bar{d}_{0}^{\prime} d_{1}^{\prime}=\bar{d}_{0} d_{1}, \quad \bar{d}_{1}^{\prime} d_{2}^{\prime}=\bar{d}_{1} d_{2}, \ldots, \bar{d}_{\mu-1}^{\prime} d_{0}^{\prime}=\bar{d}_{\mu-1} d_{0}, \text { and } \\
\bar{d}_{0}^{\prime} d_{2}^{\prime}+\bar{d}_{1}^{\prime} d_{3}^{\prime}=\bar{d}_{0} d_{2}+\bar{d}_{1} d_{3} .
\end{gathered}
$$

Since $b_{0}^{\prime}=b_{0} \omega$, i.e. $d_{0}^{\prime}=d_{0} \omega$, and $\vartheta^{\prime}=\vartheta,(25)$ implies that

$$
b_{2 k}^{\prime}=b_{2 k} \omega, \quad b_{2 k+1}^{\prime}=\frac{b_{2 k+1}}{\bar{\omega}}, \quad k=0,1,2, \ldots
$$

(26) and the last of (25) yield that $\left(|\omega|^{2}-1\right)\left(\bar{d}_{0} d_{2}-\bar{d}_{1} d_{3}|\omega|^{-2}\right)=0$, which implies (a).

Finally, if $|\omega|=1$ in (a), (26) becomes $b_{k}^{\prime}=b_{k} \omega, k=0,1,2, \ldots$, from which (b) follows immediately. This completes the proof of Property 3.

\section{Proof of LemMa 1}

Using the properties proved in paragraph 3 we prove now Lemma 1. We divide the proof into two steps. In the first step we find a partition of the set $\mathbb{N}$ of natural numbers, which satisfies only the first of the relations in (1). In the second step we refine this partition so that both relations in (1) are satisfied.

In both Steps 1 and 2 we shall need the following

Lemma 2. Let $k$ be a positive integer. If the series $S(z)$ has infinitely many limit-series, then, there are $k+1$ limit-series which satisfy the hypothesis of Property 3, i.e. for infinitely many $z$ in $E$, the $\mu$-tuples $\left(j_{1}, j_{2}, \ldots, j_{\mu}\right)$ corresponding to these $k+1$ limit-series are the same.

Proof. Let $S(z)$ have infinitely many limit-series. By Property 1 each of these limit-series has a representation of the form (13), for some positive integer $\mu$. Since $1 \leq \mu \leq N_{0}$, there is a fixed $\mu$ corresponding to infinitely many of the 
above limit-series. We consider $k \rho+1$ such limit-series, $S_{1}, S_{2}, \ldots, S_{k \rho+1}$, where $\rho$ is the number of the ordered $\mu$-tuples $\left(j_{1}, j_{2}, \ldots, j_{\mu}\right), j_{n} \in\{1,2$, $\ldots, M\}, n=1,2, \ldots, \mu$ (the $j_{n}$ 's are not necessarily distinct). Applying Property 2 to the limit-series $S_{1}$ we find an infinite subset $E_{1}$ of $E$ and an ordered $\mu$-tuple $\left(j_{11}, j_{12}, \ldots, j_{1 \mu}\right)$, such that, for every $z$ in $E_{1},(\mathrm{a}),(\mathrm{b})$, and (c) of Property 2 hold. We apply now again Property 2 to the series $S_{2}$ and we find an infinite subset $E_{2}$ of $E_{1}$ and an ordered $\mu$-tuple $\left(j_{21}, j_{22}, \ldots, j_{2 \mu}\right)$, such that, for every $z$ in $E_{2},(\mathrm{a}),(\mathrm{b})$, and (c) hold again. Continuing in the same way we obtain $k \rho+1$ infinite subsets $E_{1}, E_{2}, \ldots, E_{k \rho+1}$ of $E$ and $k \rho+1$ ordered $\mu$-tuples of indices $j_{n} \in\{1,2, \ldots, M\}, n=1,2, \ldots, \mu$. Observing that $E_{k \rho+1} \subset \cdots \subset E_{2} \subset E_{1} \subset E$ and that the number of distinct $\mu$-tuples is $\rho$, we conclude that at least $k+1$ of the limit-series, $S_{1}, S_{2}, \ldots, S_{k \rho+1}$, will correspond to the same $\mu$-tuple for every $z$ in $E_{k \rho+1}$. In other words, there are $k+1$ limit-series of $S(z)$ which satisfy the hypothesis of Property 3 and the proof is finished.

We pass now to the proof of Lemma 1.

Step 1. We shall prove the following:

The sequence $\left\{\left|a_{n}\right|\right\}$ has finitely many limit-points.

Proof. Let $\left\{\left|a_{n}\right|\right\}$ have infinitely many limit-points. Then, there are infinitely many limit-series of $S(z)$ with pairwise distinct limits $\left|b_{0}\right|=\lim _{\nu}\left|a_{\lambda_{v}}\right|$. By Lemma 2 , with $k=2$, there are three of these limit-series which satisfy the hypothesis of Property 3. Applying (a) of this property we find that the constant terms $b_{0}, b_{0}^{\prime}, b_{0}^{\prime \prime}$ of these limit-series satisfy

$$
\left|b_{0}\right|=\left|b_{0}^{\prime}\right| \text { or }\left|b_{0}^{\prime \prime}\right|=\left|b_{0}\right| \text { or }\left|b_{0}^{\prime}\right|=\left|b_{0}^{\prime \prime}\right| \text {. }
$$

This contradicts the hypothesis that the limits $\left|b_{0}\right|$ are pairwise distinct and completes the proof.

Step 2. Let now $c$ be a limit-point of the sequence $\left\{\left|a_{n}\right|\right\}$. Then, there is a subsequence $\left\{\left|a_{\lambda_{n}}\right|\right\}$ of $\left\{\left|a_{n}\right|\right\}$, such that $\lim _{n}\left|a_{\lambda_{n}}\right|=c$. Using diagonal arguments and the hypothesis that $\liminf \left|a_{n}\right|>0$, we find subsequences $\left\{\lambda_{n, j}\right\}$, $j=1,2,3, \ldots$, of $\left\{\lambda_{n}\right\}$, such that the limits $\lim _{n} a_{\lambda_{n, j}+k} / a_{\lambda_{n, j}}$ exist, for every $k=1,2,3, \ldots$, and $j=1,2,3, \ldots$.

We call $D$ the set of all sequences $\left\{q_{k}\right\}, k=1,2,3, \ldots$, for which there is a subsequence $\left\{\lambda_{\nu}\right\}$ of $\left\{\lambda_{n}\right\}$, such that

$$
\lim _{\nu} \frac{a_{\lambda_{\nu}+k}}{a_{\lambda_{\nu}}}=q_{k}, \quad k=1,2,3, \ldots
$$

We have just seen that $D \neq \varnothing$ and we note that a priori $D$ may be uncountable. We show now that $D$ is finite.

Proof. Assume $D$ infinite and let $D_{1}$ be a countable subset of $D$. Then, the elements, say $\left\{q_{k, j}\right\}, j=1,2,3, \ldots$, of $D_{1}$ are pairwise distinct sequences and, by $(27)$, for each $j=1,2,3, \ldots$, there is a subsequence $\left\{\lambda_{\nu, j}\right\}$ of $\left\{\lambda_{n}\right\}$, such that

$$
\lim _{\nu} \frac{a_{\lambda_{\nu, j}+k}}{a_{\lambda_{\nu, j}}}=q_{k, j}, \quad k=1,2,3, \ldots
$$


For each $j=1,2,3, \ldots$, we choose a subsequence of $\left\{\lambda_{\nu}, j\right\}$, which for simplicity we denote again by $\left\{\lambda_{\nu, j}\right\}$, such that $\lim _{\nu} a_{\lambda_{\nu}, j}$ exists. We call this limit $b_{0, j}$, i.e. $\lim _{\nu} a_{\lambda_{\nu, j}}=b_{0, j}$. This relation and (28) imply that $\lim _{\nu} a_{\lambda_{\nu, j}+k}=b_{0, j} q_{k, j}$, for every $k=1,2,3, \ldots$ Thus, for each $j=$ $1,2,3, \ldots$, the series

$$
b_{0, j} \sum_{k=0}^{\infty} q_{k, j} z^{k}
$$

where we have put $q_{0, j}=1$, are limit-series of $S(z)$. Since the sequences $\left\{q_{k, j}\right\}$ are pairwise distinct we have infinitely many limit-series of $S(z)$ of the form (29). Then, by Lemma 2, with $k=1$, there are two limit-series of $S(z)$, say

$$
b_{0, j} \sum_{k=0}^{\infty} q_{k, j} z^{k} \quad \text { and } \quad b_{0, \rho} \sum_{k=0}^{\infty} q_{k, \rho} z^{k},
$$

which satisfy the hypothesis of Property 3 of paragraph 3 . If now $b_{0, \rho}=b_{0, j} \omega$, then, $|\omega|=1$, since $\left|b_{0, \rho}\right|=\left|b_{0, j}\right|=c$. Thus, (b) of the above property shows that the sequences $\left\{q_{k, j}\right\}$ and $\left\{q_{k, \rho}\right\}$ are the same. This contradicts the fact that the elements of $D_{1}$ are pairwise distinct and shows that $D$ is finite.

We construct now a finite partition of $\left\{\lambda_{n}\right\}$, say $\left\{\lambda_{n, j}\right\}, j=1,2, \ldots, r$, such that, for each $j \in\{1,2, \ldots, r\}$, the limits

$$
\lim _{n} \frac{a_{\lambda_{n, j}+k}}{a_{\lambda_{n, j}}}, \quad k=1,2,3, \ldots,
$$

exist.

First, since $D$ is finite, we conclude that each one of the sequences $\left\{a_{\lambda_{n}+k} / a_{\lambda_{n}}\right\}$, $k=1,2,3, \ldots$, has finitely many limit-points.

Let $q_{1, j}, j=1,2, \ldots, r_{1}$, be the limit-points of $\left\{a_{\lambda_{n}+1} / a_{\lambda_{n}}\right\}$. Then, it is easy to see that there is a partition of $\left\{\lambda_{n}\right\}$ in $r_{1}$ subsequences, say $\left\{\lambda_{n}, j_{1}\right\}$, $j_{1}=1,2, \ldots, r_{1}$, such that

$$
\lim _{n} \frac{a_{\lambda_{n, j_{1}}+1}}{a_{\lambda_{n, j_{1}}}}=q_{1, j_{1}}, \quad j_{1}=1,2, \ldots, r_{1} .
$$

Let $q_{2, j}, j=1,2, \ldots, r_{2}$, be the limit-points of $\left\{a_{\lambda_{n}+2} / a_{\lambda_{n}}\right\}$. For each $j_{1}=1,2, \ldots, r_{1}$ we find, as above, a partition of $\left\{\lambda_{n, j_{1}}\right\}$ in a finite num-

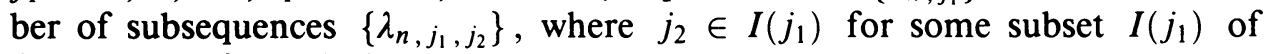
$\left\{1,2, \ldots, r_{2}\right\}$, such that

$$
\lim _{n} \frac{a_{\lambda_{n, j_{1}, j_{2}}+2}}{a_{\lambda_{n, j_{1}, j_{2}}}}=q_{2, j_{2}}
$$

In this way we have obtained a finite partition $\left\{\lambda_{n, j_{1}, j_{2}}\right\}, j_{1}=1,2, \ldots, r_{1}$, $j_{2} \in I\left(j_{1}\right)$, of $\left\{\lambda_{n}\right\}$ with the following property:

If $\left\{\lambda_{\rho}\right\},\left\{\lambda_{\rho}^{\prime}\right\}$ are any two of the sequences of this partition, then,

$$
\text { either } \lim _{\rho} \frac{a_{\lambda_{\rho}+1}}{a_{\lambda_{\rho}}} \neq \lim _{\rho} \frac{a_{\lambda_{\rho}^{\prime}+1}}{a_{\lambda_{\rho}^{\prime}}} \text { or } \lim _{\rho} \frac{a_{\lambda_{\rho}+2}}{a_{\lambda_{\rho}}} \neq \lim _{\rho} \frac{a_{\lambda_{\rho}^{\prime}+2}}{a_{\lambda_{\rho}^{\prime}}} \text {. }
$$

Since $D$ is finite if we continue in the same way this procedure must stop after a finite number of steps and thus we obtain the desired partition of the sequence $\left\{\lambda_{n}\right\}$. 
Completion of the proof of Lemma 1 . By Step 1 there are finitely many limitpoints, say $c_{j}, j=1,2, \ldots, s$, of the sequence $\left\{\left|a_{n}\right|\right\}$. To each $j$ corresponds a sequence $\left\{\lambda_{n, j}\right\}, j=1,2, \ldots, s$, and these sequences form a partition of $\mathbb{N}$, which satisfies the first of the relations (1) (see Lemma 1). We partition now each one of these sequences $\left\{\lambda_{n, j}\right\}$ as in Step 2. This way we obtain a partition of $\mathbb{N}$ in a finite number, say $m$, of sequences, such that (1) is valid and this completes the proof of Lemma 1.

\section{REMARKS AND COMMENTS}

1. V. Nestoridis proved in [5] that, under the conditions (i), (ii) and (iii) of Theorem 1 , for every $z$ in $E-\Omega=\left\{z \in C: 1-e^{i \theta} z^{m} \neq 0\right\}$, the series $S(z)$ is $(C, 1)$-summable to

$$
\sigma(z)=\frac{1}{1-e^{i \theta} z^{m}} \sum_{n=0}^{\infty}\left(a_{n}-a_{n-m} e^{i \theta}\right) z^{n},
$$

where $a_{n}=0$ if $n<0$.

Further, for every $z$ in $E-\Omega$, the set $L(z)$ is the union of $m$ concentric circles (not necessarily distinct), with common center $\sigma(z)$. The radii of these circles are given by (11), with $\mu=m$ and $d_{0}=c_{0}$,

$$
d_{\nu}=c_{\nu} \exp \left(\vartheta_{0}+\vartheta_{1}+\cdots+\vartheta_{\nu}-\nu t\right) i, \quad \nu=1,2, \ldots, m-1,
$$

where $m t=\vartheta$. We note that, following the lines of our proof of Theorem 1 , these results are consequences of Theorem 2, Property 2 and the following observations:

(a) Each limit-series of $S(z)$ is obtained from the series

$$
\left(c_{0}+c_{1} e^{i \vartheta_{0}} z+\cdots+c_{m-1} e^{i\left(\vartheta_{0}+\cdots+\vartheta_{m-2}\right)} z^{m-1}\right) \sum_{n=0}^{\infty}\left(e^{i \theta} z^{m}\right)^{n},
$$

by deleting some initial terms (if necessary) and multiplying by a suitable factor of modulus 1 (see $S\left(\left\{\lambda_{\nu}\right\}\right)$ in the proof of part (iii) of Theorem 1). Roughly speaking this means that, under the hypotheses of Theorem 1, there is "in fact" only one limit-series of $S(z)$, the series (30).

(b) Under the condition (iii) of Theorem 1, the relations (7) and $t(z)=\sigma(z)$ give the $\tau(z)+\xi(z) \sigma_{1}(z)=\sigma(z)$, i.e. $\tau(z)+\xi(z) \sigma_{1}(z)$ does not depend on $v=0,1, \ldots, m-1$ and the particular subsequence $\left\{\lambda_{\nu}\right\}$ used in the definitions of $\tau(z), \xi(z)$, and $\sigma_{1}(z)$.

We note also that the reason we cannot prove the converse of Theorem 1 from (i) and (ii) alone is the fact that $\tau(z), \xi(z)$, and $\sigma_{1}(z)$ depend on $\left\{\lambda_{\nu}\right\}$.

As it was also pointed out in [5] the angular distribution of the partial sums of $S(z)$ around $\sigma(z)$ is uniform for almost all $z$ in $E$.

2. If the set $E$ has positive Lebesgue measure, then the hypothesis that the circles $C_{j}(z), j=1,2, \ldots, M(z)$ are concentric can be omitted. Indeed, since the partial sums of $S(z)$ are bounded at every point of $E, S(z)$ is $(C, 1)$-summable, say to $\sigma(z)$, almost everywhere in $E$ (see [4, Theorem 2]) and so, by the well-known theorem of Marcinkiewicz and Zygmund mentioned in paragraph 1, for almost every $z$ in $E$ the set $L(z)$ is of circular structure with center $\sigma(z)$, i.e. $C_{j}(z)$ are concentric with common center $\sigma(z)$. 
It would be interesting to see if the hypotheses of Theorem 1 ( $E$ infinite not necessarily of positive measure) imply that the circles $C_{j}(z)$ are concentric. This would be the case if one could prove Property 1. We do not know the answer.

\section{ACKNOWLEDGMENTS}

The author wishes to thank V. Nestoridis who encouraged him to examine the case of a "finite number of circles". He also extends his thanks to S. Papadopoulou and S. Pichorides for many helpful comments on the content of this paper.

\section{REFERENCES}

1. J.-P. Kahane, Sur la structure circulaire des ensembles de points limites des sommes partielles d'une série de Taylor, Acta Sci. Math. (Szeged) 45 (1983), 247-251.

2. E. S. Katsoprinakis, On a theorem of Marcinkiewicz and Zygmund for Taylor series, Ark. Mat. 27 (1989), 105-126.

3. E. S. Katsoprinakis and V. Nestoridis, Partial sums of Taylor series on a circle, Ann. Inst. Fourier (Grenoble) 39 (1989), 715-736.

4. J. Marcinkiewicz and A. Zygmund, On the behavior of trigonometric series and power series, Trans. Amer. Math. Ser. 50 (1941), 407-453.

5. V. Nestoridis, Limit-points of partial sums of Taylor series, Matematika 38 (1991), 239-249.

6. A. Zygmund, Trigonometric series, 2nd ed. reprinted, vols. I, II, Cambridge Univ. Press, 1979.

Department of Mathematics, University of Crete, P. O. Box 1470, Iraklion (Crete), GREECE

E-mail address: katsopr@talos.cc.uch.gr 